\title{
Functionalization of Boron-Doped Nanocrystalline Diamond with N3 Dye Molecules
}

W.S. Yeap, X. Liu, D. Bevk, A. Pasquarelli, L. Lutsen, Mats Fahlman, W. Maes and K. Haenen

\section{Linköping University Post Print}

\section{Tweet}

N.B.: When citing this work, cite the original article.

Original Publication:

W.S. Yeap, X. Liu, D. Bevk, A. Pasquarelli, L. Lutsen, Mats Fahlman, W. Maes and K. Haenen, Functionalization of Boron-Doped Nanocrystalline Diamond with N3 Dye Molecules, 2014, ACS Applied Materials and Interfaces, (6), 13, 10322-10329.

http://dx.doi.org/10.1021/am501783b

Copyright: American Chemical Society http://pubs.acs.org/

Postprint available at: Linköping University Electronic Press

http://urn.kb.se/resolve?urn=urn:nbn:se:liu:diva-109373 


\title{
Functionalization of boron-doped nanocrystalline diamond with N3 dye molecules
}

\section{W.S. Yeap,${ }^{1 *}$ X. Liu, ${ }^{2 \ddagger}$ D. Bevk,,${ }^{1,3}$ A. Pasquarelli, ${ }^{4}$ L. Lutsen, ${ }^{3}$ M. Fahlman, ${ }^{2}$ W. Maes, ${ }^{1,3}$ and K.} Haenen ${ }^{1,3 *}$

\footnotetext{
${ }^{1}$ Hasselt University, Institute for Materials Research (IMO), B-3590 Diepenbeek, Belgium

${ }^{2}$ Linköping University, Department of Physics, Chemistry and Biology, S-58183 Linköping, Sweden

${ }^{3}$ IMEC vzw, IMOMEC, B-3590 Diepenbeek, Belgium

${ }^{4}$ Ulm University, Institute of Electron Devices and Circuits, 89069 Ulm, Germany

* To whom correspondence should be addressed.
}

Tel. : +321126 8826; fax: +3211268899

E-mail address: wengsiang.yeap@uhasselt.be (W.S. Yeap)

E-mail address: ken.haenen@uhasselt.be (K. Haenen)

₹ These authors contributed equally.

\begin{abstract}
N3 dye molecules [cis-bis(isothiocyanato)-bis(2,2'-bipyridyl-4,4'-dicarboxylato)-ruthenium(II)] are covalently attached onto boron-doped nanocrystalline diamond (B:NCD) thin films through a combination of coupling chemistries, i.e. diazonium, Suzuki and EDC-NHS. X-ray and ultraviolet photoelectron spectroscopy (XPS and UPS), and near-edge X-ray absorption fine structure spectroscopy (NEXAFS) are used to verify the covalent bonding of the dye on the B:NCD surface (as compared to a hydrogen-terminated reference). The spectroscopic results confirm the presence of a dense N3 chromophore layer and the positions of the frontier orbitals of the dye relative to the band edge of the B:NCD thin film are inferred as well. Proof of concept photoelectrochemical measurements show a strong increase in photocurrent as compared to nondye-functionalized B:NCD films. This study opens up the possibility to apply N3-sensitized B:NCD thin films as hole conductors in dye-sensitized solar cells.
\end{abstract}

Keywords: boron-doped diamond, diazonium coupling, Suzuki cross-coupling, N3 dye, spectroscopic surface characterization, photocurrent 


\section{Introduction}

Diamond is an extraordinary material with excellent properties such as high optical transparency, corrosive resistance, a wide electrochemical potential window and biocompatibility. With recent advanced technologies such as microwave plasma-enhanced chemical vapor deposition (MW PECVD), nanocrystalline diamond (NCD) thin films can be grown on various substrates at lower temperatures and pressures and reduced costs from cheap hydrocarbon precursors. ${ }^{1,2}$ Furthermore, the films can show p-type conductivity by doping with boron. High boron doping ratios afford metallic conductivity with a specific resistivity as low as $0.005 \Omega \mathrm{cm}$. This has opened up various technological applications for boron-doped nanocrystalline diamond (B:NCD) thin films. They have for instance been used as efficient transducers for sensors. ${ }^{3-7}$ B:NCD thin film electrodes decorated with metal nanoparticles have shown catalytic effects in the reduction of $\mathrm{CO}_{2}$ and alkenes, ${ }^{8}$ the oxidation of methanol, ${ }^{9}$ and the degradation of environmental pollutants. ${ }^{10}$ Recently, boron-doped diamond nanoparticles have been produced from CVD diamond films. ${ }^{11}$ These nanoparticles not only are conductive due to boron doping but also have high surface-to-volume ratio owing to their very small size. This new interesting diamond material may be applied for a broad range of applications. Despite these advantages, the major downside of diamond is the low reactivity of its surface, which makes the direct attachment of functional organic molecules quite challenging. This difficulty can be overcome by using photochemical attachment techniques, ${ }^{12}$ diazonium coupling, ${ }^{13-15}$ or plasma treatment. ${ }^{16}$ Post-modification of the diamond surface with (other) organic moieties can then be carried out employing various efficient chemical reactions. ${ }^{14,15,17-19}$

The first report on dye sensitization of p-type semiconductive diamond electrodes was published in 1999 by Fujishima et al. ${ }^{20}$ Photocurrent was observed when a synthetic semiconductive p-type diamond electrode was photosensitized by the $\mathrm{Ru}(\mathrm{bpy})_{3}{ }^{2+}$ (bpy $=2,2$ '-bipyridine) molecule in an electrolyte solution. More recently, robust covalent linking of $\mathrm{Ru}(\mathrm{tpy})_{2}$ (tpy = 2,2':6',2"'terpyridine) dye molecules onto a boron-doped diamond (BDD) surface has been demonstrated. ${ }^{18}$ The electronic structure of the obtained films was systematically probed by spectroscopic techniques. ${ }^{21}$ The HOMO level of the Ru(tpy $)_{2}$ dye was found to be $0.9 \mathrm{eV}$ lower than the valence band maximum (VBM) of BDD, prompting the authors to suggest a possible facile hole transfer 
from $\mathrm{Ru}(\mathrm{tpy})_{2}$ to $\mathrm{BDD}$. This result could pave the way to employ dye-sensitized BDD as an electrode in dye-sensitized solar cells (DSSCs).

The N3 dye [cis-bis(isothiocyanato)-bis(2,2'-bipyridyl-4,4'-dicarboxylato)-ruthenium(II)] (Figure 1) is one of the most efficient sensitizers used in DSSCs. ${ }^{21}$ The dye has a large molar extinction coefficient in the visible region and is more widely used than $\mathrm{Ru}(\mathrm{tpy})_{2}$ and $\mathrm{Ru}(\mathrm{bpy})_{3}{ }^{2+}$. Sensitization of the N3 dye with n-type semiconductors has been reported before. ${ }^{22-25}$ Dyesensitized tandem solar cells have also been made using p-type semiconductors such as $p$-NiO in combination with N3 dye molecules. This p-type semiconductor co-deposited onto titania acted both as a light absorber and hole conductor. ${ }^{26} \mathrm{~B}$ :NCD possesses several advantages over NiO. $\mathrm{B}: \mathrm{NCD}$ is highly transparent ${ }^{27}$ in the visible range, whereas $\mathrm{NiO}$ absorbs a significant amount of light. ${ }^{28} \mathrm{NiO}$ also has a lower hole mobility $\left(4 \times 10^{-8} \mathrm{~cm}^{2} / \mathrm{s}\right)^{29}$ as compared to B:NCD $(0.77$ $\left.\mathrm{cm}^{2} / \mathrm{s}\right){ }^{30} \mathrm{~B}: \mathrm{NCD}$ is known to be very chemically stable as well. ${ }^{1}$ In DSSCs, p-doped diamond electrodes might be employed as hole conductors to replace the traditional highly corrosive triiodide electrolyte. $^{21}$ Moreover, as shown previously by Zhong et al., ${ }^{14}$ a highly stable photocurrent conversion could be observed for molecular dye-functionalized B:NCD, which was attributed to strong interfacial $\mathrm{C}-\mathrm{C}$ covalent bonding. To the best of our knowledge, covalent attachment of the N3 dye on p-doped B:NCD thin films has not been performed so far. To obtain insights whether B:NCD could be a possible hole conductor when sensitized with N3 molecules, first of all covalent attachment of the N3 dye on a B:NCD surface has to be established.

Herein we report a strategy to covalently attach the $\mathrm{N} 3$ dye on $\mathrm{B}: \mathrm{NCD}$ thin films using a combination of coupling chemistries. The B:NCD surface is first functionalized with 4bromophenyl groups by diazonium coupling. Suzuki cross-coupling is then applied to couple 4aminophenylboronic pinacol ester to the bromophenyl moieties to obtain (1,1'-biphenyl)-4-amine groups on the B:NCD surface, and finally the amine functions are used to attach the N3 dye through EDC-NHS coupling. Wet chemical functionalization in solution is preferred here over physical plasma treatment to obtain a more homogeneous surface. Moreover, the use of this method can easily be extended to functionalized particles, including the nano-sized boron-doped diamond mentioned above. ${ }^{11}$ After each functionalization step the surface composition is analyzed by X-ray photoelectron spectroscopy (XPS). Ultraviolet photoelectron spectroscopy (UPS) and near-edge X-ray absorption fine structure spectroscopy (NEXAFS) are used to 
investigate the HOMO and LUMO levels, respectively, of the N3 dye relative to the band edges of diamond. Photoelectrochemical measurements are performed to evaluate photocurrent generation by the functionalized B:NCD films. The results obtained might open up new possibilities in solar energy research by demonstrating the suitability of dye-sensitized B:NCD thin films as hole conductors in DSSCs.

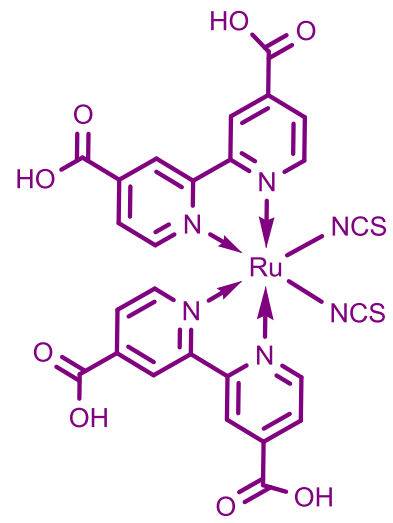

Figure 1. Chemical structure of the N3 dye.

\section{Experimental}

\subsection{Chemical Reagents}

Commercially available chemicals were purchased in the purest grades available and used without further purification. $N$-hydroxysuccinimide (NHS), cesium acetate, palladium(II) acetate, 4-aminophenylboronic acid pinacol ester, 4-bromobenzenediazonium tetrafluoroborate, methyl viologen dichloride hydrate, sodium sulphate and tri(o-tolyl)phosphine were purchased from Sigma-Aldrich. The N3 dye was purchased from DYESOL. 1-Ethyl-3-[3(dimethylamino)propyl]carbodiimide hydrochloride (EDC) was purchased from Thermo Scientific. All solvents used for reactions and rinsing were of HPLC grade, unless otherwise stated. Water used for reactions and rinsing was prepared with a Type 1 Ultrapure water by Sartorius stedium Biotech. 


\subsection{Diamond Growth}

Heavily boron-doped nanocrystalline diamond thin films $(150 \mathrm{~nm})$ were grown by microwave plasma-enhanced chemical vapor deposition from methane/hydrogen mixtures $\left(1 \% \mathrm{CH}_{4}\right)$ in an ASTeX 6500 reactor. The substrates used were $1 \times 1 \mathrm{~cm}$ fused silica. The growth conditions were as follows: substrate temperature $700-900{ }^{\circ} \mathrm{C}$, total gas flow $500 \mathrm{sccm}$, total pressure in the reactor 30 Torr, microwave power $3500 \mathrm{~W}$. Trimethylborane gas was added during the growth with a ratio of $10000 \mathrm{ppm} \mathrm{B/C}$ (with respect to $\mathrm{CH}_{4}$ ) to ensure good electrical conductivity. ${ }^{30}$ Sheet resistance (Rs), as measured by a four-point probe measurement, was $207 \Omega / \mathrm{sq}$, which agrees well with the typical value for boron-doped NCD grown at this conditions. ${ }^{27,30}$ Prior to the diamond growth, the fused silica substrates were cleaned for 15 min each in RCA $1\left(30 \% \mathrm{NH}_{3}+\right.$ $\left.30 \% \mathrm{H}_{2} \mathrm{O}_{2}+\mathrm{H}_{2} \mathrm{O} ; 1: 1: 5\right)$ and $\mathrm{RCA} 2\left(37 \% \mathrm{HCl}+30 \% \mathrm{H}_{2} \mathrm{O}_{2}+\mathrm{H}_{2} \mathrm{O} ; 1: 1: 5\right)$ solutions at $90{ }^{\circ} \mathrm{C}$. Following this, the cleaned fused silica substrates were seeded with nanodiamond powder in water to improve the nucleation density. After deposition, the diamond samples were allowed to cool down in the reactor for 30 min under vacuum. To remove any graphitic layers, the asdeposited diamond films were boiled in an acid mixture of $99 \% \mathrm{H}_{2} \mathrm{SO}_{4}+30 \% \mathrm{HNO}_{3}(3: 1)$ at $90{ }^{\circ} \mathrm{C}$ for $30 \mathrm{~min}$. After rinsing and sonicating with plenty of deionized water, the diamond samples were subjected to hydrogenation. Figure 2 displays a scanning electron microscopy (SEM) image of a typical as-grown B:NCD thin film.

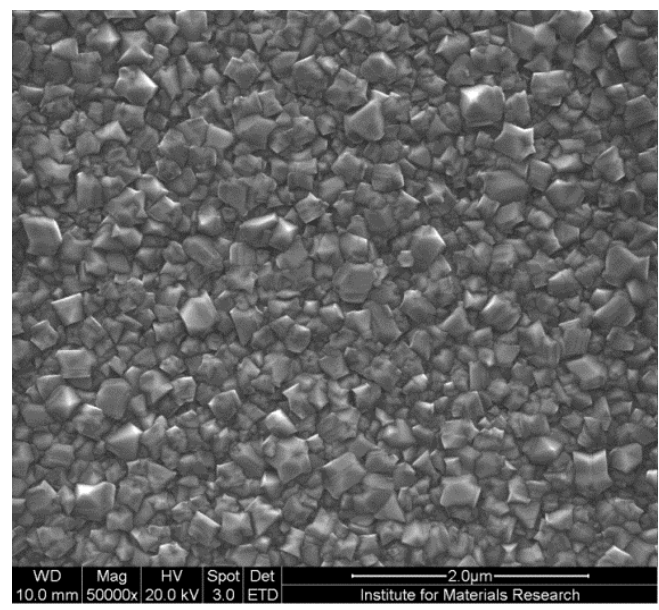

Figure 2. SEM image of a typical as-grown B:NCD thin film. 


\subsection{Hydrogenation}

Hydrogenation was performed using the same PECVD reactor (ASTeX 6500). To create a hydrogen-terminated surface, all diamond samples were treated with hydrogen plasma under the following conditions: i. $500 \mathrm{sccm}$ of hydrogen flux, 30 Torr reactor pressure with $3500 \mathrm{~W}$ microwave power for $2 \mathrm{~min}$; ii. $500 \mathrm{sccm}$ of hydrogen flux, 15 Torr reactor pressure with $2500 \mathrm{~W}$ microwave power for $5 \mathrm{~min}$. At the end of the plasma treatment, the microwave power was switched off and the samples were allowed to cool down under hydrogen flux for $40 \mathrm{~min}$.

\subsection{Diamond Surface Functionalization}

Functionalization of the diamond surface with 4-bromophenyl groups was carried out by simply immersing the hydrogenated B:NCD samples into a solution of 4-bromobenzenediazonium tetrafluoroborate $(5 \mathrm{mM})$ in $0.1 \mathrm{M} \mathrm{HCl}$ for $120 \mathrm{~min} .{ }^{14}$ After surface modification, the substrates were sequentially rinsed and sonicated in ultrapure water, THF and $n$-hexane to remove any physisorbed molecules.

All preparations for the Suzuki cross-coupling reactions were performed in a glovebox. A $15 \mathrm{~mL}$ ACE pressure tube (Sigma Aldrich) containing a magnetic stirring bar and 4-bromophenylfunctionalized B:NCD was charged with 4-aminophenylboronic acid pinacol ester ( $20 \mathrm{mg}, 100$ $\mu \mathrm{mol}), 10 \mathrm{~mol} \%$ tri $(o$-tolyl $)$ phosphine $(3.1 \mathrm{mg}, 10 \mu \mathrm{mol})$, cesium acetate $(19 \mathrm{mg}, 100 \mu \mathrm{mol}), 5$ mol\% palladium(II) acetate $(1.1 \mathrm{mg}, 5 \mu \mathrm{mol})$, and $5 \mathrm{~mL}$ of THF. The resulting mixture was then heated at $80{ }^{\circ} \mathrm{C}$ for $18 \mathrm{~h}$. After the reaction, the substrates were sequentially rinsed and sonicated in methanol, THF and $n$-hexane to remove any physisorbed molecules.

The N3 dye was coupled onto the (1,1'-biphenyl)-4-amine-functionalized B:NCD films through EDC-NHS coupling. To this extent, the $\mathrm{N} 3$ dye $(37 \mathrm{mg}, 50 \mu \mathrm{mol})$ was dissolved in $2 \mathrm{~mL}$ of absolute ethanol. In a separate vial, EDC (38 mg, $200 \mu \mathrm{mol})$ and NHS (58 mg, $500 \mu \mathrm{mol})$ were dissolved in $3 \mathrm{~mL}$ of ultrapure water. The resulting two solutions were mixed together. The (1,1'biphenyl)-4-amine-functionalized B:NCD films were then submerged into this solution for $5 \mathrm{~h}$ at room temperature. After the reaction, the functionalized diamond samples were sequentially rinsed and sonicated in ethanol and water to remove any physisorbed molecules. 


\subsection{Photoelectron Spectroscopy}

Photoemission experiments were carried out using a Scienta ESCA 200 spectrometer in ultrahigh vacuum with a base pressure of $1 \times 10^{-10}$ mbar. The measurement chamber is equipped with a monochromatic $\mathrm{Al} \mathrm{K \alpha} \mathrm{X}$-ray source and He discharged lamp providing photons with $1486.6 \mathrm{eV}$ for XPS and $21.22 \mathrm{eV}$ for UPS, respectively. The XPS experimental conditions were set as such that the full width at half maximum of the clean $\mathrm{Au} 4 \mathrm{f}_{7 / 2}$ line is $0.65 \mathrm{eV}$. The total-energy resolution of the measurements in UPS, determined by the Fermi edge of clean gold, is about 0.1 $\mathrm{eV}$. All spectra were measured at a photoelectron take-off angle of $0^{\circ}$ normal emission. The UPS spectra have been corrected for the contributions from He I satellites radiation.

\subsection{Near-Edge X-ray Absorption Fine Structure Spectroscopy (NEXAFS)}

Polarized X-ray absorption spectroscopy measurements were performed at the beam line D1011 of the MAX-II storage ring, located at the MAX-Laboratory for Synchrotron Radiation Research in Lund, Sweden. The energy resolution was about $100 \mathrm{meV}$ at photon energy close to the $\mathrm{C}-\mathrm{K}$ edge. The absorption spectrum was collected in the total electron yield mode by measuring the sample current. Simultaneously, the absorption spectrum with partial electron yield was also recorded by a multi-channeltron plate (MCP) detector with a bias of $-100 \mathrm{~V}$ to screen the electrons with lower kinetic energy. The raw data have been corrected for the energy dependence of the incident X-ray beam and subsequently normalized to have the same absorption edge step well above the threshold.

\subsection{Photoelectrochemical Measurements}

Photoelectrochemical measurements were performed in a $\mathrm{N}_{2}$-saturated $0.1 \mathrm{M} \mathrm{Na}_{2} \mathrm{SO}_{4}$ solution containing $5 \mathrm{mM}$ methyl viologen $\left(\mathrm{MV}^{2+}\right)$ as an electron carrier in a three-electrode glass cell containing B:NCD as a working electrode, a Pt counter electrode and an $\mathrm{Ag} / \mathrm{AgCl}$ reference electrode. The glass cell was placed in a dark Faraday cage and controlled by a potentiostat (Princeton Applied Research PARSTAT 2273). The cold light light source employed was a 150 W halogen lamp (Mitutoyo Fiber Optic Illuminator). The incident light intensity was focused and calibrated with a photodiode to $150 \mathrm{~mW} / \mathrm{cm}^{2}$. The area of the B:NCD samples exposed to light was $0.07 \mathrm{~cm}^{2}$. 


\section{Results and Discussion}

\subsection{Chemical Functionalization Strategies}

The various steps in the chemical functionalization protocol of the B:NCD films are shown in Scheme 1. The combination of diazonium, Suzuki, and EDC-NHS coupling reactions provides a powerful chemical route to covalently immobilize N3 dye molecules onto a B:NCD surface. After each step, the success of the coupling reaction was monitored by XPS (vide infra).

(a) Diazonium coupling

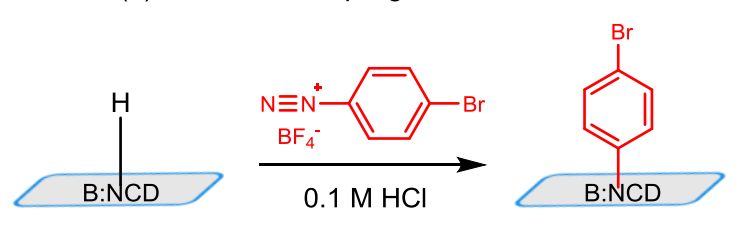

(b) Suzuki cross-coupling
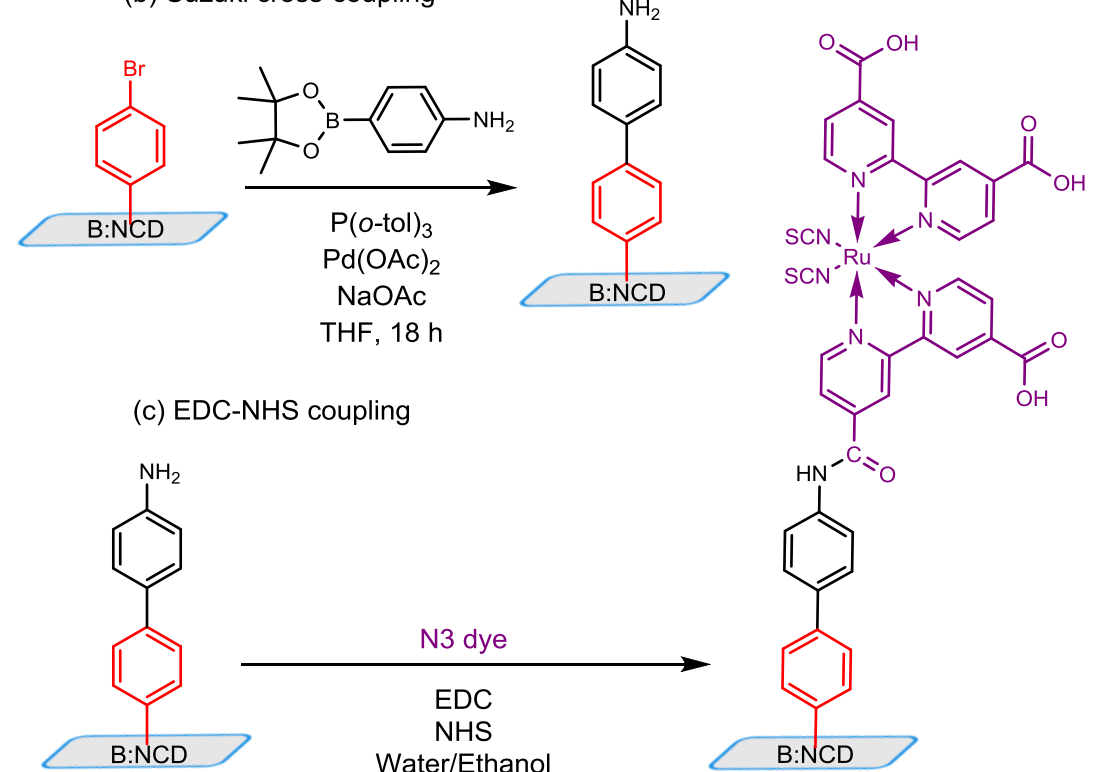

-NHS coupling
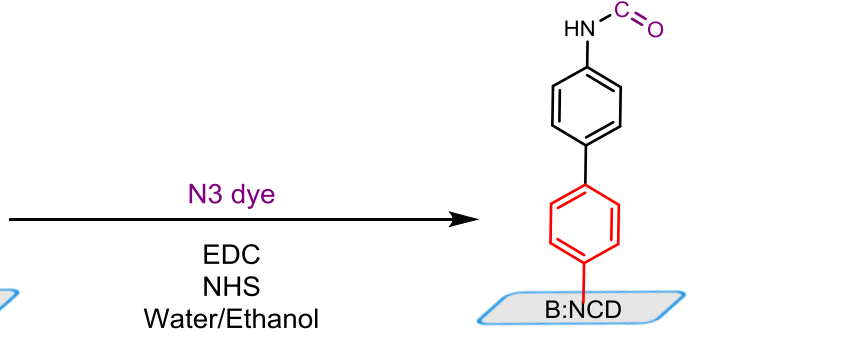

Scheme 1. Subsequent coupling chemistries employed for the functionalization of B:NCD with N3 dyes.

The surface modification sequence starts with functionalization of the B:NCD surface with 4bromophenyl groups. These entities can be covalently attached on hydrogenated B:NCD through reaction with the respective diazonium salt. This is done by submerging a hydrogenated B:NCD 
film into an acidic solution of 4-bromobenzenediazonium tetrafluoroborate for 2 hours. Previous studies have already shown that diazonium salts can be coupled spontaneously to hydrogenated diamond surfaces due to direct charge transfer between the hydrogenated diamond - with negative electron affinity - and the diazonium cations. ${ }^{14}$ Spontaneous coupling provides a better control of the thickness of the organic layer compared to electrochemical grafting. ${ }^{31}$ A monolayer of 4-bromophenyl groups can be attained by spontaneous coupling. ${ }^{14}$

The bromoaryl-functionalized diamond film can then react further via Suzuki reactions. ${ }^{14,17}$ Suzuki-Miyaura cross-coupling of 4-aminophenylboronic acid pinacol ester to the prefunctionalized B:NCD creates a layer of (1,1'-biphenyl)-4-amine groups on the B:NCD surface. The selection of an efficient catalytic system is of great importance to achieve high coupling yields in transition metal catalyzed cross-couplings. ${ }^{32,33}$ Best results were obtained for a $\mathrm{Pd}(\mathrm{OAc})_{2}-\mathrm{P}(o \text {-tol })_{3}$ catalytic system compared to other systems using $\mathrm{Pd}\left(\mathrm{PPh}_{3}\right)_{4}$ or $\mathrm{Pd}(\mathrm{dppf}) \mathrm{Cl}_{2}$. Finally, EDC-NHS coupling of the N3 dye to the (1,1'-biphenyl)-4-amine groups was carried out in an ethanol/water mixture.

\subsection{Surface Composition by X-ray Photoelectron Spectroscopy}

After each functionalization step, the variation of the surface components is a key parameter to assess the success of the functionalization process. XPS is a powerful tool to probe the surface composition of materials. Figure 3a shows the XPS Br 3p peaks of 4-bromophenyl-functionalized B:NCD. The appearance of the Br 3p signals at 181 and $189 \mathrm{eV}$ clearly indicates that the B:NCD surface is decorated with $\mathrm{Br}$ due to 4-bromobenzene functionalization. ${ }^{14}$ Furthermore, a $\left(1,1^{\prime}-\right.$ biphenyl)-4-amine layer forms after Suzuki coupling of 4-aminophenylboronic acid pinacol ester. Successful cross-coupling was confirmed through the corresponding N 1s spectra, as shown in Figure $3 \mathrm{~b}$. The $\mathrm{N} 1 \mathrm{~s}$ spectrum of the amino groups of (1,1'-biphenyl)-4-amine-functionalized $\mathrm{B}: \mathrm{NCD}$ exhibits a peak at $400 \mathrm{eV}$, which is in agreement with earlier reports. ${ }^{31}$ 
(a)

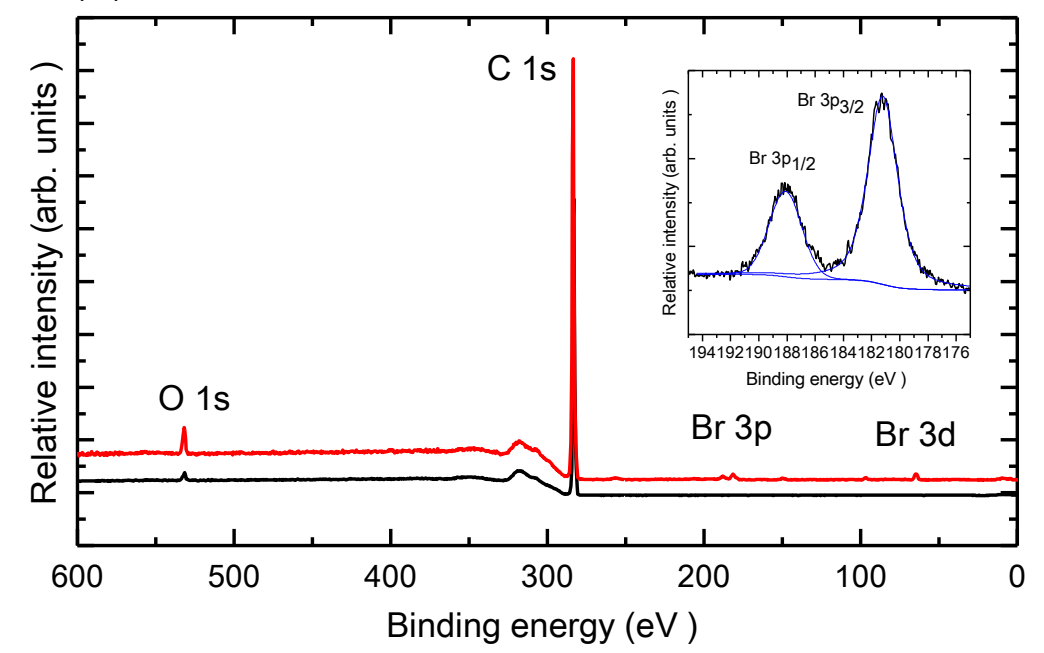

(b)

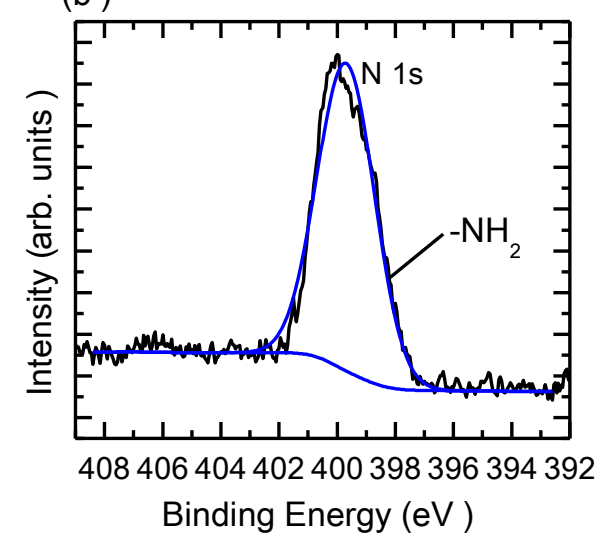

Figure 3. (a) XPS survey spectra of a 4-bromophenyl-functionalized B:NCD surface (red line) as compared to a reference sample of hydrogenated B:NCD (black line). Inset: High-resolution $\mathrm{Br}$ 3p XPS spectrum of 4-bromophenyl-functionalized B:NCD. (b) High-resolution N 1s XPS spectrum of (1,1'-biphenyl)-4-amine-functionalized B:NCD.

Upon final functionalization with the N3 chromophore, new XPS spectral features clearly appear, as indicated in Figure 4, consistent with the chemical structure of the dye. The XPS peak at 281 $\mathrm{eV}$, which corresponds to $\mathrm{Ru} 3 \mathrm{~d}_{5 / 2}$, can be considered as a fingerprint of the $\mathrm{N} 3$ dye, since each dye molecule introduces one $\mathrm{Ru}$ atom. ${ }^{35}$ Furthermore, the other elements in the N3 dye with different chemical environment are also clearly demonstrated in the XPS results. ${ }^{36}$ For instance, there are two kinds of nitrogen species in the N3 dye, one from the four pyridine groups and another one due to the two isothiocyanate groups. ${ }^{35}$ The splitting of the $\mathrm{N} 1$ s peak can be clearly observed, with two components included in the $\mathrm{N}$ 1s spectral feature. The shoulder at lower 
binding energy $(399 \mathrm{eV})$ can be assigned to the $\mathrm{N}=\mathrm{C}=\mathrm{S}$ groups,${ }^{35,37}$ whereas the sharp peak at higher binding energy $(400 \mathrm{eV})$ can be attributed to the four pyridine units. ${ }^{35,36}$ Due to the additional contribution to the $\mathrm{N}$ 1s signal from the amide linkage to the $\mathrm{N} 3$ dye, the spectral weight ratio of the components at higher and lower binding energy is slightly different from the ratio observed in the pure N3 dye. Furthermore, the sulfur atoms of the isothiocyanate groups are also observed at $163 \mathrm{eV}$ (S 2p peak). ${ }^{35,36}$ The observed broad O 1s peak is due to several oxygen species in the N3 dye and surface adsorption of oxygen from the environment before XPS characterization.
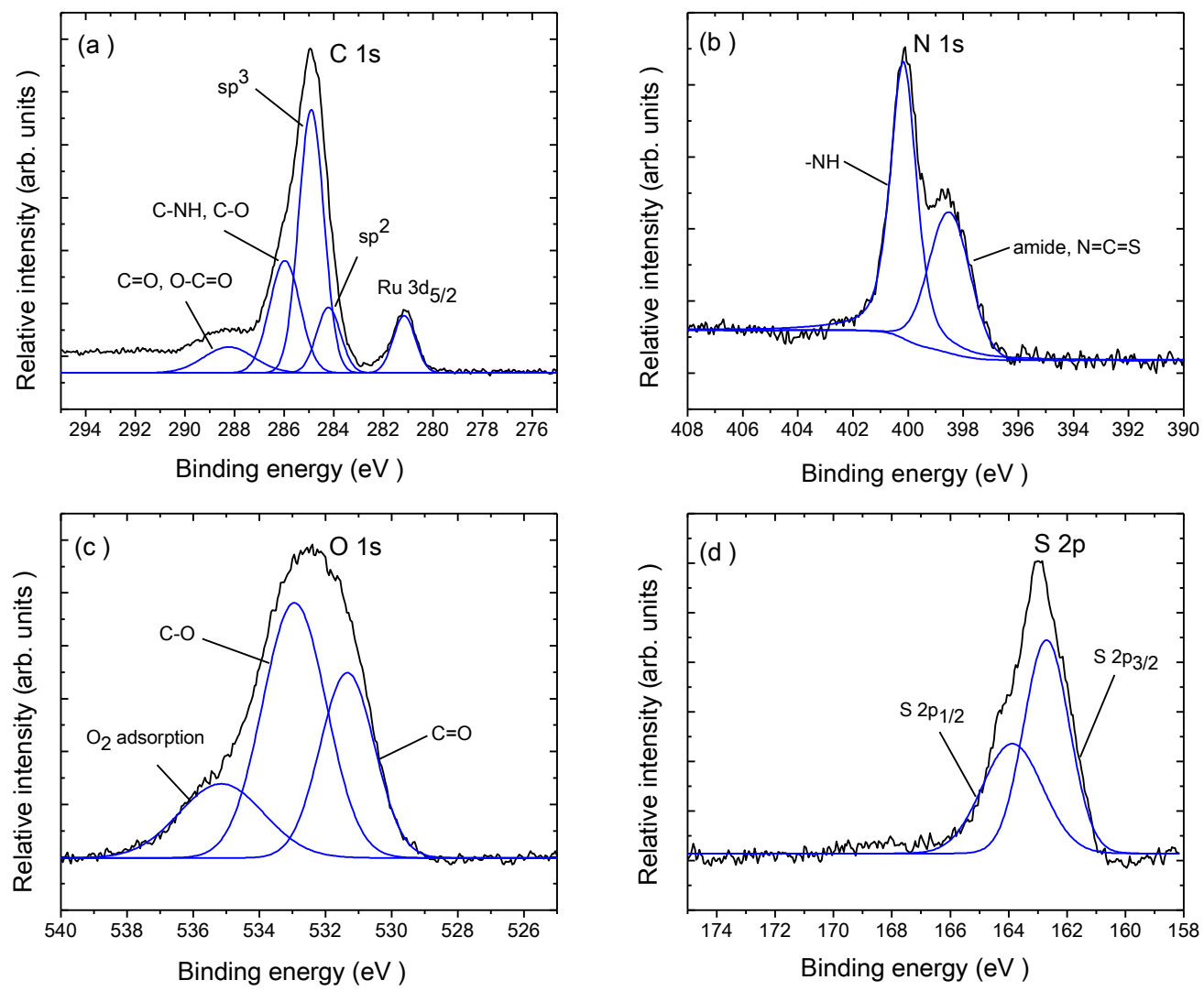

Figure 4. XPS spectra after EDC-NHS coupling of the N3 dye to (1,1'-biphenyl)-4-aminefunctionalized B:NCD: (a) C 1s, (b) N 1s, (c) O 1s, and (d) S 2p core level spectra.

The coverage of the $\mathrm{N} 3$ dye on $\mathrm{B}: \mathrm{NCD}$ after functionalization is crucial for future device applications. The number of $\mathrm{N} 3$ dye molecules on the B:NCD surface can be deduced from the XPS results by the areal Ru density. The advantage of using the Ru intensity is that the change of spectral weight is considerably less influenced by impurities and/or contaminants on the 
functionalized B:NCD thin films compared to other relevant elements such as C, O, and S. Based on the core-level $\mathrm{C}$ 1s spectrum with the $\mathrm{Ru} 3 \mathrm{~d}$ peak at $281.1 \mathrm{eV}$, and considering the following assumptions: ${ }^{19}$ 1) The $\mathrm{Ru} 3 \mathrm{~d}$ photoelectrons at the topmost surface do not experience scattering. 2) Carbon from the initial layer and from underlying NCD can be treated as being equivalent in the contribution of the $\mathrm{C}$ 1s signal. 3) The inelastic mean free path of the $\mathrm{C}$ 1s photoelectron is $2 \mathrm{~nm}$. 4) The number density of carbon atoms in NCD is $1.66 \times 10^{23}$ atoms $/ \mathrm{cm}^{3}$. 5) The areal density of carbon is $3.32 \times 10^{16}$ atoms $/ \mathrm{cm}^{2}$ (NCD mass density $\sim 3.3 \mathrm{~g} / \mathrm{cm}^{3}$ ), the XPS area ratio can be expressed as:

$$
\frac{\mathrm{A}_{\mathrm{Ru} 3 \mathrm{~d}_{5}}}{\mathrm{~A}_{\mathrm{C} 1 \mathrm{~s}}}=\frac{\mathrm{Ru}\left(\frac{\text { atoms }}{\mathrm{cm}^{2}}\right) * \mathrm{~S}_{\mathrm{Ru} 3 \mathrm{~d}_{5}}}{\mathrm{C}\left(\frac{\text { atoms }^{2}}{\mathrm{~cm}^{2}}\right) * \mathrm{~S}_{\mathrm{C} 1 \mathrm{~s}}}
$$

Taking into account the atomic sensitivity factors of $\mathrm{Ru} 3 \mathrm{~d}_{5 / 2}$ and $\mathrm{C} 1 \mathrm{~s}$ and the spectral weight of $\mathrm{C} 1 \mathrm{~s}$ and $\mathrm{Ru} 3 \mathrm{~d}$, the surface coverage of $\mathrm{N} 3$ dyes on the B:NCD thin films was determined to be $3 \times 10^{14} \mathrm{Ru} / \mathrm{cm}^{2}$. This corresponds to $3 \mathrm{Ru} / \mathrm{nm}^{2}$, which indicates that the $\mathrm{N} 3$ dye forms a quite dense film on the B:NCD surface.

\subsection{Ultraviolet Photoelectron Spectroscopy (UPS)}

UPS is a powerful tool to directly probe the electronic properties of the valence band of materials. Figure 5 illustrates the UPS spectrum of N3 dye-functionalized B:NCD compared to the pure N3 dye. It can clearly be seen that the functionalized B:NCD film reproduces the spectral features of the pure chromophore. When the UPS intensity is plotted on a logarithmic scale, the weak valence band feature can be clearly distinguished. The first valence band peak appears around 2.0 $\mathrm{eV}$ in both the functionalized and pure samples. The second peak at $\sim 4 \mathrm{eV}$ was only observed in the functionalized diamond sample, which could be due to the interaction between the dye and the B:NCD substrate. 

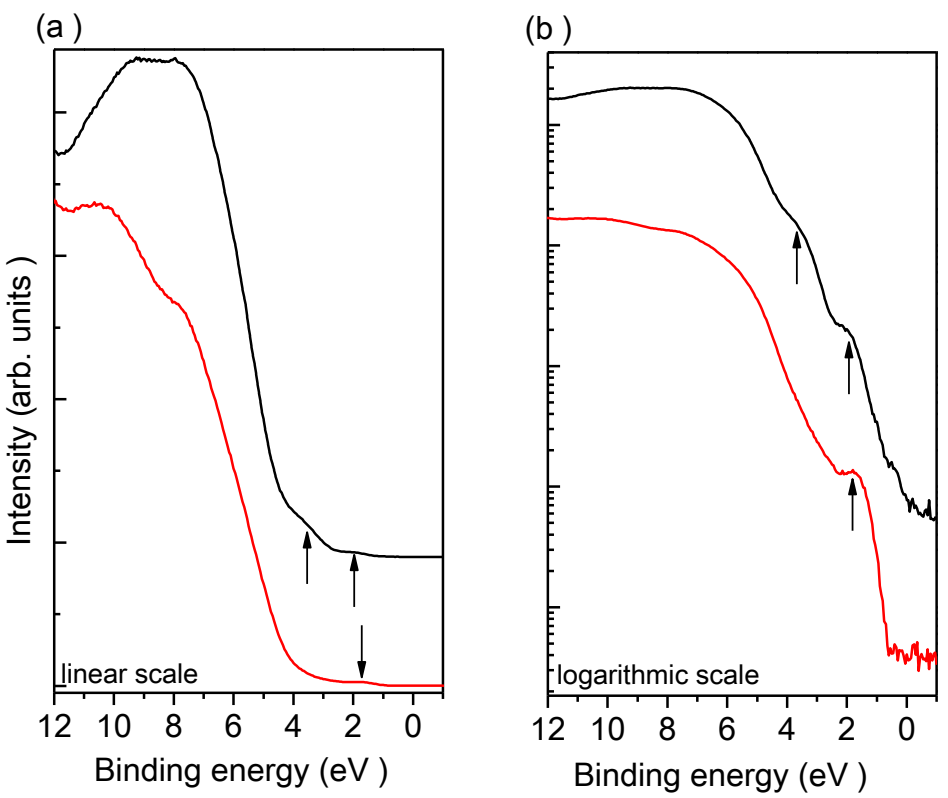

Figure 5. Comparison of the UPS spectra of N3 dye-functionalized B:NCD (black line) and the pure N3 dye (drop-casted on a Au substrate) (red line) on a linear (a) and logarithmic (b) scale.

To determine the HOMO energy level of the N3 chromophore, a UPS spectrum of a B:NCD film functionalized with N3 dye molecules was taken (Figure 6). The HOMO of the dye is compared to a similar spectrum of a hydrogen-terminated B:NCD thin film. The HOMO level of the N3 dye emerges at $2.0 \mathrm{eV}$ below the Fermi level. Moreover, by linear extrapolation of the hydrogenterminated B:NCD thin film UPS data, the position of the VBM of diamond with respect to its Fermi level can be estimated. It is approximately $1.5 \mathrm{eV}$ below the (surface) Fermi level. Accordingly, the HOMO of the N3 dye lies $0.5 \mathrm{eV}$ below the VBM of B:NCD (Figure 6). These energy differences have implications on the hole transport between the B:NCD thin film and the appended N3 dye. Based on the principle of charge transfer in DSSCs, upon photogeneration of an exciton, the electron is transferred to an electron acceptor while the hole is transferred from the donor to the working electrode film. Hence, a good match between the HOMO of the donor molecule and the valence band of the electrode is important for efficient hole injection. For $\mathrm{B}: \mathrm{NCD}$, the VBM is situated rather close $(0.5 \mathrm{eV})$ to the HOMO of the functionalized $\mathrm{N} 3$ dye, which should favor hole injection from the dye to the B:NCD thin film. ${ }^{17,21}$ 


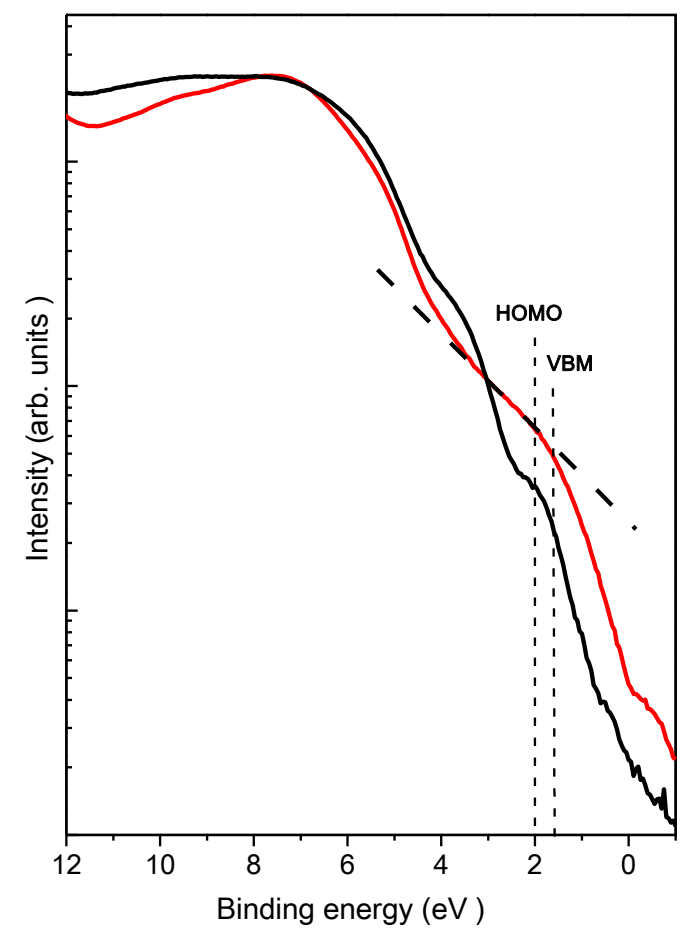

Figure 6. Comparison of the UPS spectra of the N3 dye-functionalized B:NCD (black line) and a bare hydrogen-terminated B:NCD film (red line). Linear extrapolation of the valence band edge yields an estimate of the VBM the of B:NCD thin film. The HOMO of the N3 dye lies $0.5 \mathrm{eV}$ below the VBM.

\subsection{Near-Edge X-ray Absorption Fine Structure Spectroscopy (NEXAFS)}

When we turn to the empty states of the N3 dye-functionalized B:NCD film, as obtained from NEXAFS measurements (Figure 7), it can be seen that there are some clear spectral features in the $\mathrm{C}$ 1s absorption spectrum. There are two distinguished peaks around $285 \mathrm{eV}$, a shoulder at $\sim 287 \mathrm{eV}$, and another three peaks up to a photon energy of $290 \mathrm{eV}$. As indicated by the XPS data, the N3 dye can form a film on the B:NCD surface with high coverage. The spectral features related to the B:NCD thin film are hence completely suppressed. On the other hand, when compared with the NEXAFS spectrum of the pure N3 dye, the influence of the B:NCD thin film upon the spectral features of the empty states of the N3 molecule can be extracted. The spectral features related to the N3 dye appear at higher photon energy, for instance between 287 and 290 eV (Figure 7). All absorption peaks are at approximately the same position in both the functionalized B:NCD sample and the pure N3 dye. A significant change was observed on the 
empty $\pi^{*}$ states at lower photon energy, i.e. around $285 \mathrm{eV}$. The LUMO and LUMO+1 energy levels of the pure N3 dye are observed at around 284.1 and $285.1 \mathrm{eV}$, respectively, and they are shifted to 284.4 and $284.9 \mathrm{eV}$ for the functionalized B:NCD sample. The change of the LUMO and LUMO+1 positions shows the influence of B:NCD on the functionalized dye on top. ${ }^{21}$ To ensure that the (1,1'-biphenyl)-4-amine linker does not significantly distort the result, the spectrum of (1,1'-biphenyl)-4-amine-functionalized B:NCD was taken as well. It contains a single $\pi^{*}$ peak at $285 \mathrm{eV}$ originating from the biphenyl layer covalently attached onto B:NCD. This peak partially fills the valley between the $\pi^{*}$ peaks of the N3 dye, without strongly affecting their peak positions.

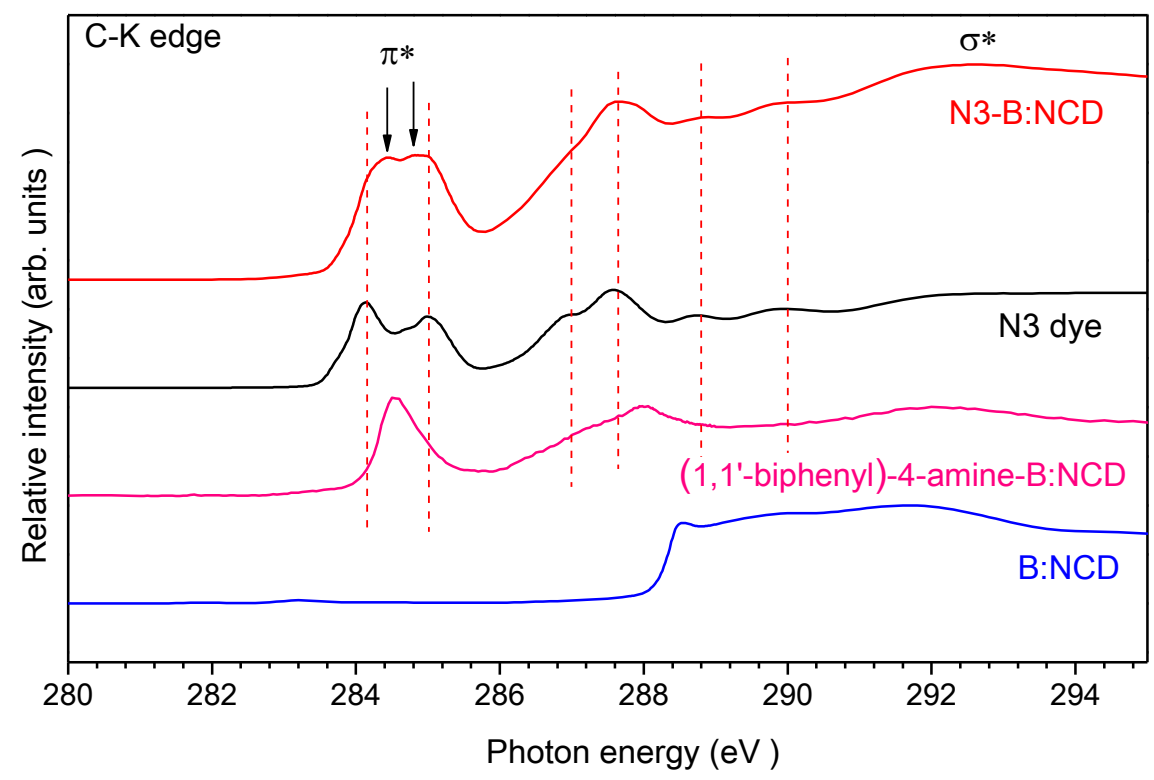

Figure 7. Comparison of the C-K edge NEXAFS spectra of N3-functionalized B:NCD, the pure N3 dye, (1,1'-biphenyl)-4-amine-functionalized B:NCD and a hydrogen-terminated B:NCD film.

\subsection{Photoelectrochemical Measurements}

As a proof of concept, photoelectrochemical measurements were performed using either a hydrogen-terminated B:NCD, a (1,1'-biphenyl)-4-amine-functionalized B:NCD or a N3functionalized B:NCD film as the working electrode in a typical three-electrode electrochemical system. After equilibration in darkness, the light source was switched on and off approximately every $30 \mathrm{~s}$ for three cycles. The response curves show a tremendously increased photocurrent 
intensity for the N3-functionalized B:NCD electrode at $-0.1 \mathrm{~V}$ bias as compared to both control systems (Figure 8). This agrees well with the mechanism that, upon light generation of electronhole pairs in the $\mathrm{N} 3$ dye, the separated electrons flow toward the $\mathrm{MV}^{2+}$ (methyl viologen) electron carrier and the holes toward the B:NCD electrode (at negative bias). ${ }^{14,17}$

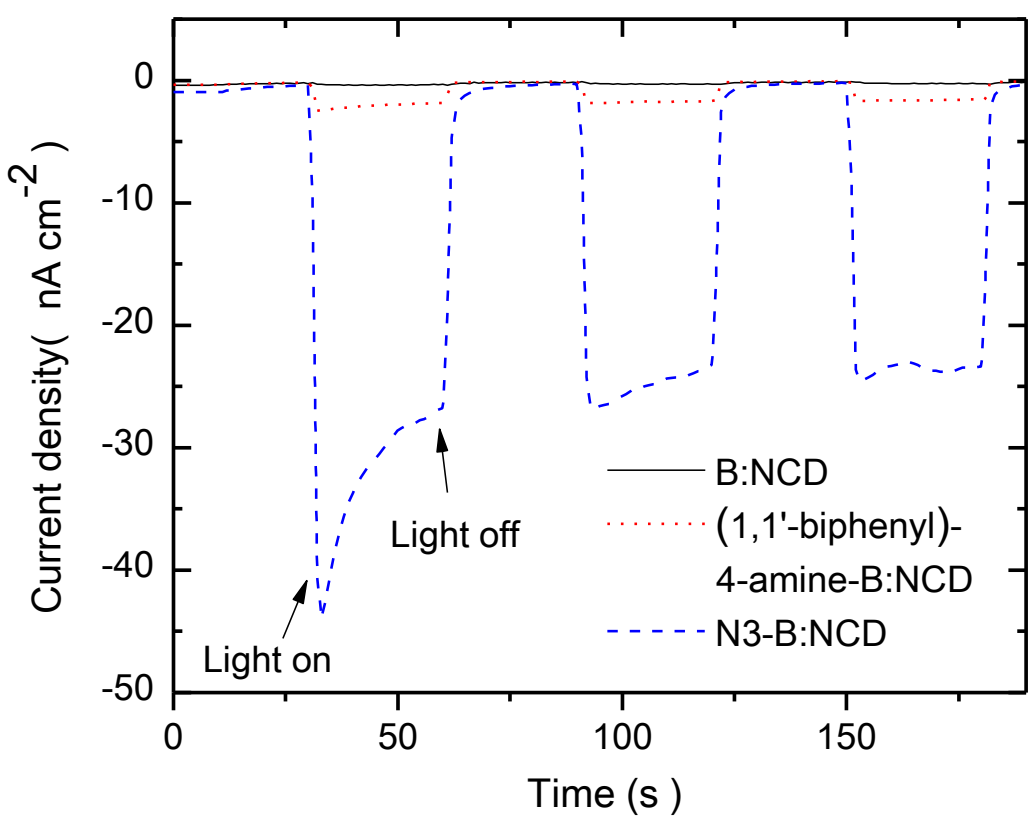

Figure 8. Photocurrent response of B:NCD (black solid line), (1,1'-biphenyl)-4-amine-B:NCD (red dotted-line) and N3-functionalized B:NCD (blue dashed-line) electrodes in a $5 \mathrm{mM}$ methyl viologen solution (in $0.1 \mathrm{M} \mathrm{Na}_{2} \mathrm{SO}_{4}$ ) at $-0.1 \mathrm{~V}$ bias ( $\mathrm{vs} \mathrm{Ag} / \mathrm{AgCl}$ ).

\section{Conclusions}

In conclusion, we have shown that N3 dye molecules can be covalently attached onto borondoped nanocrystalline diamond thin films. Calculations based upon the $\mathrm{Ru}$ presence as analyzed by XPS indicate that a dense layer of chromophore molecules $\left(\sim 3 \times 10^{14} \mathrm{Ru} / \mathrm{cm}^{2}\right)$ is achieved. UPS and NEXAFS measurements reveal that there is an interaction between the B:NCD thin film and the functionalized N3 dye. As evidenced by UPS measurements - matching of the HOMO level of the $\mathrm{N} 3$ dye with the VBM of the B:NCD thin film - holes generated during photoexcitation can possibly transfer from the dye to the B:NCD thin film, which was confirmed by proof of concept photocurrent measurements. This opens up a pathway toward the application of diamond- 
based dye-sensitized solar energy conversion via systematic energy level alignment. Efforts in this direction are currently ongoing within our groups.

\section{Acknowledgements}

This work was financially supported by the Special Research Fund of Hasselt University, the Research Foundation Flanders (FWO) (G.0555.10N), and the EU FP7 Collaborative Project "MOLESOL" (No. 256617). X. L. acknowledges support from The Swedish Research Council Linnaeus grant LiLi-NFM. We thank P. Robaeys for the four-point probe measurements and Prof. J. D'Haen for the SEM image.

\section{References}

1. Williams, O. A.; Nesladek, M.; Daenen, M.; Michaelson, S.; Hoffman, A.; Osawa, E.; Haenen, K.; Jackman, R. B. Growth, Electronic Properties and Applications of Nanodiamond. Diam. Relat. Mater. 2008, 17, 1080-1088.

2. Potocky, S.; Kromka, A.; Potmesil, J.; Remes, Z.; Vorlicek, V.; Vanecek, M.; Michalka, M. Investigation of Nanocrystalline Diamond Films Grown on Silicon and Glass at Substrate Temperature Below 400 Degrees C. Diam. Relat. Mater. 2007, 16, 744-747.

3. Reitinger, A. A.; Hutter, N. A.; Donner, A.; Steenackers, M.; Williams, O. A.; Stutzmann, M.; Jordan, R.; Garrido, J. A. Functional Polymer Brushes on Diamond as a Platform for Immobilization and Electrical Wiring of Biomolecules. Adv. Funct. Mater. 2013, 23, 2979-2986.

4. Ishii, Y.; Ivandini, T. A.; Murata, K.; Einaga, Y. Development of Electrolyte-Free Ozone Sensors Using Boron-Doped Diamond Electrodes. Anal. Chem. 2013, 85, 4284-4288.

5. Subramanian, P.; Motorina, A.; Yeap, W. S.; Haenen, K.; Coffinier, Y.; Zaitsev, V.; Niedziolka-Jonsson, J.; Boukherroub, R., Szunerits, S. An Impedimetric Immunosensor Based on Diamond Nanowires Decorated with Nickel Nanoparticles. Analyst 2014, 139, 1726-1731. 
6. Kondo, T.; Horitani, M.; Sakamoto, H.; Shitanda, I.; Hoshi, Y.; Itagaki, M.; Yuasa, M. Screenprinted Modified Diamond Electrode for Glucose Detection. Chem. Lett. 2013, 42, 352-354.

7. Wang, Q.; Subramanian, P.; Li, M. S.; Yeap, W. S.; Haenen, K.; Coffinier, Y.; Boukherroub, R.; Szunerits, S. Non-enzymatic Glucose Sensing on Long and Short Diamond Nanowire Electrodes. Electrochem. Commun. 2013, 34, 286-290.

8. Dhakshinamoorthy, A.; Navalon, S.; Sempere, D.; Alvaro, M.; Garcia, H. Reduction of Alkenes Catalyzed by Copper Nanoparticles Supported on Diamond Nanoparticles. Chem. Commun. 2013, 49, 2359-2361.

9. Hutton, L. A.; Vidotti, M.; Patel, A. N.; Newton, M. E.; Unwin, P. R.; Macpherson, J. V. Electrodeposition of Nickel Hydroxide Nanoparticles on Boron-Doped Diamond Electrodes for Oxidative Electrocatalysis. J. Phys. Chem. C 2011, 115, 1649-1658.

10. Toghill, K. E.; Wildgoose, G. G.; Moshar, A.; Mulcahy, C.; Compton, R. G. The Fabrication and Characterization of a Bismuth Nanoparticle Modified Boron Doped Diamond Electrode and Its Application to the Simultaneous Determination of Cadmium(II) and Lead(II). Electroanal. 2008, 20, 1731-1737.

11. Heyer, S.; Janssen, W.; Turner, S.; Lu, Y.; Yeap, W. S.; Verbeek, J.; Haenen, K.; Krueger, K., Toward Deep Blue Nano Hope Diamonds: Heavily Boron-Doped Diamond Nanoparticles. ACS Nano 2014, DOI: 10.1021/nn500573x.

12. Wang, X. Y.; Landis, E. C.; Franking, R.; Hamers, R. J. Surface Chemistry for Stable and Smart Molecular and Biomolecular Interfaces via Photochemical Grafting of Alkenes. Acc. Chem. Res. 2010, 43, 1205-1215.

13. Jian, W.; Firestone, M. A.; Auciello, O.; Carlisle, J. A. Surface Functionalization of Ultrananocrystalline Diamond Films by Electrochemical Reduction of Aryldiazonium Salts. Langmuir 2004, 20, 11450-11456.

14. Zhong, Y. L.; Loh, K. P.; Midya, A.; Chen, Z. K. Suzuki Coupling of Aryl Organics on Diamond. Chem. Mater. 2008, 20, 3137-3144. 
15. Zhong, Y. L.; Ng, W.; Yang, J. X.; Loh, K. P. Electrostatically Self-Assembled Polyoxometalates on Molecular-Dye-Functionalized Diamond. J. Am. Chem. Soc. 2009, 131, $18293-18298$.

16. Nakamura, T.; Ohana, T. Surface Functionalization of Diamond Films by Photoreaction of Elemental Sulfur and Their Surface Properties. Jpn. J. Appl. Phys. 2012, 51, 085201-085204.

17. Zhong, Y. L.; Midya, A.; Ng, Z.; Chen, Z. K.; Daenen, M.; Nesladek, M.; Loh, K. P. Diamond-Based Molecular Platform for Photoelectrochemistry. J. Am. Chem. Soc. 2008, 130, $17218-17219$.

18. Yao, S. A.; Ruther, R. E.; Zhang, L. H.; Franking, R. A.; Hamers, R. J.; Berry, J. F. Covalent Attachment of Catalyst Molecules to Conductive Diamond: $\mathrm{CO}_{2}$ Reduction Using "Smart" Electrodes. J. Am. Chem. Soc. 2012, 134, 15632-15635.

19. Ruther, R. E.; Rigsby, M. L.; Gerken, J. B.; Hogendoorn, S. R.; Landis, E. C.; Stahl, S. S.; Hamers, R. J. Highly Stable Redox-Active Molecular Layers by Covalent Grafting to Conductive Diamond. J. Am. Chem. Soc. 2011, 133, 5692-5694.

20. Nakabayashi, S.; Ohta, N.; Fujishima, A. Dye Sensitization of Synthetic p-type Diamond Electrode. Phys. Chem. Chem. Phys. 1999, 1, 3993-3997.

21. Zegkinoglou, I.; Cook, P. L.; Johnson, P. S.; Yang, W. L.; Guo, J. H.; Pickup, D.; GonzalezMoreno, R.; Rogero, C.; Ruther, R. E.; Rigsby, M. L.; Ortega, J. E.; Hamers, R. J.; Himpsel, F. J. Electronic Structure of Diamond Surfaces Functionalized by Ru(tpy) 2 . J. Phys. Chem. C 2012, $116,13877-13883$.

22. Grätzel, M. Photoelectrochemical Cells. Nature 2001, 414, 338-344.

23. Ling, T.; Song, J. G.; Chen, X. Y.; Yang, J.; Qiao, S. Z.; Du, X. W. Comparison of ZnO and $\mathrm{TiO}_{2}$ Nanowires for Photoanode of Dye-Sensitized Solar Cells. J. Alloy Compd. 2013, 546, 307313.

24. Mali, S. S.; Betty, C. A.; Bhosale, P. N.; Patil, P. S. Eosin-Y and N3-Dye Sensitized Solar Cells (DSSCs) Based on Novel Nanocoral $\mathrm{TiO}_{2}$ : A Comparative Study. Electrochim. Acta 2012, $59,113-120$. 
25. Mane, R. S.; Pathan, H. M.; Lokhande, C. D.; Han, S. H. An Effective Use of Nanocrystalline CdO Thin Films in Dye-Sensitized Solar Cells. Sol. Energy 2006, 80, 185-190.

26. Kalyanasundaram, K. Photochemical and Photoelectrochemical Approaches to Energy Conversion. In Dye-Sensitized Solar Cells; Kalyanasundaram, K., Eds.; EPFL Press: Lausanne, 2010; pp 1-43.

27. Lim, C. H. Y. X.; Zhong, Y. L.; Janssens, S.; Nesladek, M.; Loh, K. P. Oxygen-Terminated Nanocrystalline Diamond Film as an Efficient Anode in Photovoltaics. Adv. Funct. Mater. 2010, 20, 1313-1318.

28. Nattestad, A.; Mozer, A. J.; Fischer, M. K. R.; Cheng, Y. B.; Mishra, A.; Bauerle, P.; Bach, U. Highly Efficient Photocathodes for Dye-Sensitized Tandem Solar Cells. Nat. Mater. 2010, 9, 3135.

29. Mori, S.; Fukuda, S.; Sumikura, S.; Takeda, Y.; Tamaki, Y.; Suzuki, E.; Abe, T. ChargeTransfer Process in Dye-Sensitized NiO Solar Cells. J. Phys. Chem. C 2008, 112, 16134-16139.

30. Janssens, S. D.; Pobedinskas, P.; Vacik, J.; Petrakova, V.; Ruttens, B.; D'Haen, J.; Nesladek, M.; Haenen, K.; Wagner, P. Separation of Intra- and Intergranular Magnetotransport Properties in Nanocrystalline Diamond Films on The Metallic Side of The Metal-Insulator Transition. New J. Phys. 2011, 13, 083008-083024.

31. Lud, S. Q.; Steenackers, M.; Jordan, R.; Bruno, P.; Gruen, D. M.; Feulner, P.; Garrido, J. A.; Stutzmann, M. Chemical Grafting of Biphenyl Self-Assembled Monolayers On Ultrananocrystalline Diamond. J. Am. Chem. Soc. 2006, 128, 16884-16891.

32. Shen, W. Palladium Catalyzed Coupling of Aryl Chlorides with Arylboronic Acids. Tetrahedron Lett. 1997, 38, 5575-5578.

33. Barder, T. E.; Walker, S. D.; Martinelli, J. R.; Buchwald, S. L. Catalysts for Suzuki-Miyaura Coupling Processes: Scope and Studies of The Effect of Ligand Structure. J. Am. Chem. Soc. 2005, 127, 4685-4696. 
34. Eck, W.; Stadler, V.; Geyer, W.; Zharnikov, M.; Golzhauser, A.; Grunze, M. Generation of Surface Amino Groups On Aromatic Self-Assembled Monolayers By Low Energy Electron Beams - A First Step Towards Chemical Lithography. Adv. Mater. 2000, 12, 805-808.

35. Syres, K. L.; Thomas, A. G.; Cant, D. J. H.; Hardman, S. J. O.; Preobrajenski, A. Pyrocatechol As A Surface Capping Molecule On Raffle $\mathrm{TiO}_{2}$ (110). Surf. Sci. 2012, 606, 273277.

36. Terasaki, N.; Nitahara, S.; Akiyama, T.; Yamada, S. Structural Characterization and Photocurrent Properties of cis-di(thiocyanato)-bis(4,40-dicarboxy-2,20-bipyridine) Ruthenium(II) Monolayers on the Gold Surfaces. Jpn. J. Appl. Phys. 2005, 44, 2795-2798.

37. Srinivasan, V.; Walton, R. A. X-ray Photoelectron Spectra of Inorganic Molecules: XX. Observations Concerning the Sulfur 2p Binding Energies in Metal Complexes of Thiourea. Inorg. Chim. Acta 1977, 25, L85-L86. 